\title{
Applying a sensor energy supply communication scheme to big data opportunistic networks
}

\author{
Zhigang CHEN ${ }^{1}$ and Jia WU ${ }^{1}$ \\ ${ }^{1}$ School of Software, Central South University \\ Chang Sha, Hu Nan, 410075 - Chima \\ [e-mail: czg@mail.csu.edu.cn] \\ [e-mail: jiawu5110@163.com] \\ *Corresponding author: Jia WU
}

Received November 4, 2015; revised January 18, 2015; revised February 28, 2016; accepted April 3, 2016; published May 31, 2016

\begin{abstract}
Energy consumption is an important index in mobile ad hoc networks. Data packet transmission increases among nodes, particularly in big data communication. However, nodes may be unable to transmit data packets because of energy over-consumption. Consequently, information may be lost and network communication may block. While opportunistic network is a kind of mobile ad hoc networks, researchers do not focus on energy consumption in opportunistic network communication. This study proposed an effective sensor energy supply scheme that can be applied in opportunistic networks. This scheme considers nodes sensor requests and communication model. In this scheme, nodes do not only accomplish energy supply in communication, but also extend communication time in opportunistic networks. Compared with the Spray and Wait algorithm and the Binary Spray and Wait algorithm in simulations, the proposed scheme extends communication time, increases data packet transmission, and accomplishes energy supply among nodes.
\end{abstract}

Keywords: sensor, opportunistic network, communication, big data, energy supply

This work was supported in part by the National Natural Science Foundation of China(61379057, 61309001, 61272149) ; Doctoral Fund of Ministry of Education of China(20120162130008); Innovation Foundation For Postgraduate(2014zzts043) 


\section{Introduction}

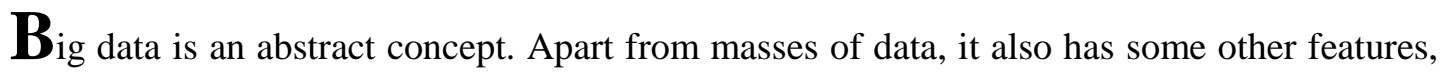
which determine the difference between itself and"massive data"or"very big data”. At present, although the importance of big data has been generally recognized, people still have different opinions on its definition.

In general, big data shall mean the data sets that could not be perceived, acquired, managed, and processed by traditional IT and software/hardware tools within a tolerable time. Because of different concerns, scientific and technological enterprises, research scholars, data analysts, and technical practitioners have different definitions of big data. The following definitions may help us have a better understanding on the profound social, economic, and technological connotations of big data. Big data communication is a challenge. Because there are no clearly transmission destination nodes, the process of transmission is complex and a great number of nodes must be taken part in. That means more network resources must be used, more complex transmission model should be established.

In 2014, process Estrin suggests that in big data research, many activity locus are generated with a great number of mobile devices when people use theirs on net. Some individuation data are extracted from activity locus can discover and judge human activity. These data are named "small data" [19]. From small data, researches can establish some parts of big data researching model to solve many problems in big data environment.

In big data communication, opportunistic network is a type of self-organized wireless sensor network [1][2]. This new-generation sensor network is based on the development of delay tolerant networks [3][4][5]. It involves the information deliver process between nodes, which does not require a complete path. Instead, information moves between nodes, and the communication area is formatted to realize this process [6][11]. During the entire process, information is transmitted to the next hop node via the "carry-store-forward" approach [7][8].

One advantage of this model is its flexible information transmission process; moreover, it does not require creating numerous links [9]. This model can transfer information between nodes conveniently and efficiently, which is appropriate in a big data environment. However, researchers who have studied opportunistic networks seldom pay attention to the effect of energy consumption on networks. Nodes have to transmit huge amounts of data packets, particularly in a big data environment, which accelerates the consumption of energy. If energy supply fails while transferring data packets, the nodes that are more involved in the transmission will die immediately. By contrast, the nodes that participate less in the transmission will retain more energy, but will lose the opportunity to gain data packets. As a representative algorithm in opportunistic networks, Epidemic algorithm [9] is inapplicable to such networks because of its excessive flooding, which results in extremely high energy consumption. Spray and Wait algorithm [10] is developed from the foundation of the Epidemic algorithm. The Spray and Wait algorithm transmits a copy of data to the neighbor node to reduce over-flooding by spraying the source node. Nonetheless, nodes transfer more data in big data environment, which can also lead to excessive energy consumption. 
To solve the aforementioned problem, we consider the following aspects:

(1) the possibility of providing energy supply for the source node,

(2) whether energy is supplied by a neighbor node,

(3) and how to assign energy supply to neighbor nodes.

In response to the issue of the energy consumption of nodes, this study proposes a sensor request model as research object. An energy transmission and supply scheme in opportunistic networks is established. In this scheme, Sensor Request Energy Supply Algorithm (SRESA) based on energy supply and equilibrium is proposed. This algorithm can flexibly transfer data packets. In addition, this model can establish energy supply, equilibrium energy distribution among nodes, and extend life time with nodes in communication.

\section{Related Work}

At present, studies on opportunistic networks have mainly focused on routing algorithms. Such algorithms can be ported to different application fields and scenes to establish interdisciplinary research by improving available algorithms. Some existing opportunistic network algorithms are discussed as follows.

Aliotta et al. mentioned Direct Delivery algorithm in Ref. [12]. This algorithm forwards information when a source node meets destination nodes, which minimizes the cost of the entire network space. However, the process of transferring nodes involves significant transmission delay and deliver ratio is reduced to the lowest value.

Lokhov et al. presented Epidemic algorithm in Ref. [13] that is based on the forwarding mechanism similar to that of infectious diseases. In this algorithm, when two nodes meet, they exchange messages that the other does not store. This process is adopted to XOR and ensures that the two nodes obtain additional information. A sufficient network bandwidth, buffer space, and other resources can guarantee that routes from source node transferring information to destination node are the shortest. In practice, however, congestion can occur as the number of network nodes involved in the transmission of network information increases. Consequently, a considerable amount of energy is consumed because the relevant resources of the actual network nodes are limited. This method is used in numerous real environments and typically cannot obtain a good effect because limited resources.

In Ref. [14], Wang et al. proposed Spray and Wait algorithm, which was combined with the characteristics of the infectious disease algorithm. The proposed algorithm is divided into the spray phase and the wait phase. In the former, the source node initially counts the nodes that can transmit information around it. It then forwards its information to neighbor nodes by spraying. In the latter, data are transmitted to the target node via direct delivery to complete the transfer process if no transfer node is found during the spray phase. This method has improved over-flooding in the original Epidemic algorithm. However, the spray phase may also waste the resources of the source node when too many neighbor nodes are involved because of over consumption. Therefore, this algorithm will engender the death of nodes in some strongly random networks because of excessive spraying.

Spyropoulos et al. proposed PRoPHET algorithm in Ref. [15]. They conducted a probabilistic statistical computation of object nodes that could be passed. Then, suitable transfer nodes were estimated to form data grouping, which improved the utilization of a network. In Ref. [16], Leguay et al. proposed the MV algorithm based on probability. This 
algorithm calculates forwarding probability through the statistics of historical records when nodes meet and visit a communication area.

Burgess et al. presented MaxProp algorithm based on priority setting in Ref. [17]. In this algorithm, deliver queue is determined by setting the priority of arrays when two nodes meet. In this manner, the excessive consumption of resources can be reduced. This algorithm also ensures the queue of the delivery object while simultaneously improving efficiency. A self-organizing delivery area is formed within the space to realize selected communication between nodes.

In Ref. [18], Burns et al. proposed Context-aware routing algorithm to calculate the deliver probability of reaching the target node. By computing periodic transfer probability, intermediate nodes are obtained. Along with data collection and data packet, information is transmitted directly to the node with a high transfer probability.

Based on the analyses and summaries of several existing works, we demonstrate the energy problem in a model of opportunistic networks by combining its characteristics in this study.

\section{Design of sensor energy supply communication scheme}

In opportunistic networks, communication between nodes requires consumption energy. Given that some nodes carry considerable amounts of useful information, energy consumption increases when such nodes send messages to their neighbor nodes. By contrast, some nodes carry less information or do not carry effective information at all, which causes them to consume less energy. This condition results in the predicament wherein the energy of valuable nodes is consumed too fast, while that of invaluable nodes is not. Each source node has to send its packets to its neighbors, particularly in Spray and Wait algorithm. Although the algorithm can do well in information coverage, the excessive energy consumption of active nodes with a considerable amount of information is likely to cause their death. If these those aspects can be addressed, then we can realize the energy transfer and supply of nodes in opportunistic networks, equilibrium their carrying energy, and maintain network performance.

\subsection{Model environment}

To describe and analyze the model, the model environment is designed as follows:

1) Network $G(V, E)$ is constituted by $N$ nodes, $G$ is undirected connected graph, $V$ is node, and $E$ is link path.

2) When nodes $u$ and $v$ transmit messages, the link is $(u, v) \quad$. $€$ Egoes both ways.

3) At least one relevance node and route exists in network $G$. The relevance node and route are deleted. $G$ is divided to several subnetworks, which are undirected connected graphs.

4) The cache space of each node is limited. Both nodes have the same cache space, which is enough for messages storage and transmission.

5) The nodes in the network are mobile and the pattern is random. The speed of establishing relevance and transmission is considerably larger than the speed of node movement.

6) The nodes appear regularly when they have moved for a long time. According to history meeting, the next hop node can be calculated and predicted. 


\subsection{Design of energy supply between the nodes}

In wireless networks, energy which can be supplied between neighbors have been applied in the real time and the real world. It likes in sensor wireless networks [20] and vehicular networks [21]. They complete energy supply by node movement and meeting. Effective algorithm in such networks can achieve this target.

The Spray and Wait algorithm [10] has the advantage of completing a full coverage of the transmission information of nodes in opportunistic networks. It increases the energy consumption of nodes, particularly in a big data environment. However, energy over-consumption can easily cause the death of nodes. Therefore, an energy supply scheme should be introduced into the model of the Spray and Wait algorithm.

The Binary Spray and Wait algorithm [11] is an optimization of the Spray and Wait algorithm. It reduces routing overhead in the method by making the source node send $1 / 2$ data packets to neighbor nodes and saving the rest of the data. This method can transmit data packets efficiently and reduce energy consumption. However, this algorithm can be adopted only in networks with few nodes. With the increase in the number of nodes and data packets, the problems in the Spray and Wait algorithm reoccur in big data communication networks.

To solve big data transmission and energy consumption problems, a sensor request selection of data packets and energy supply model should be implemented in sensor opportunistic networks.

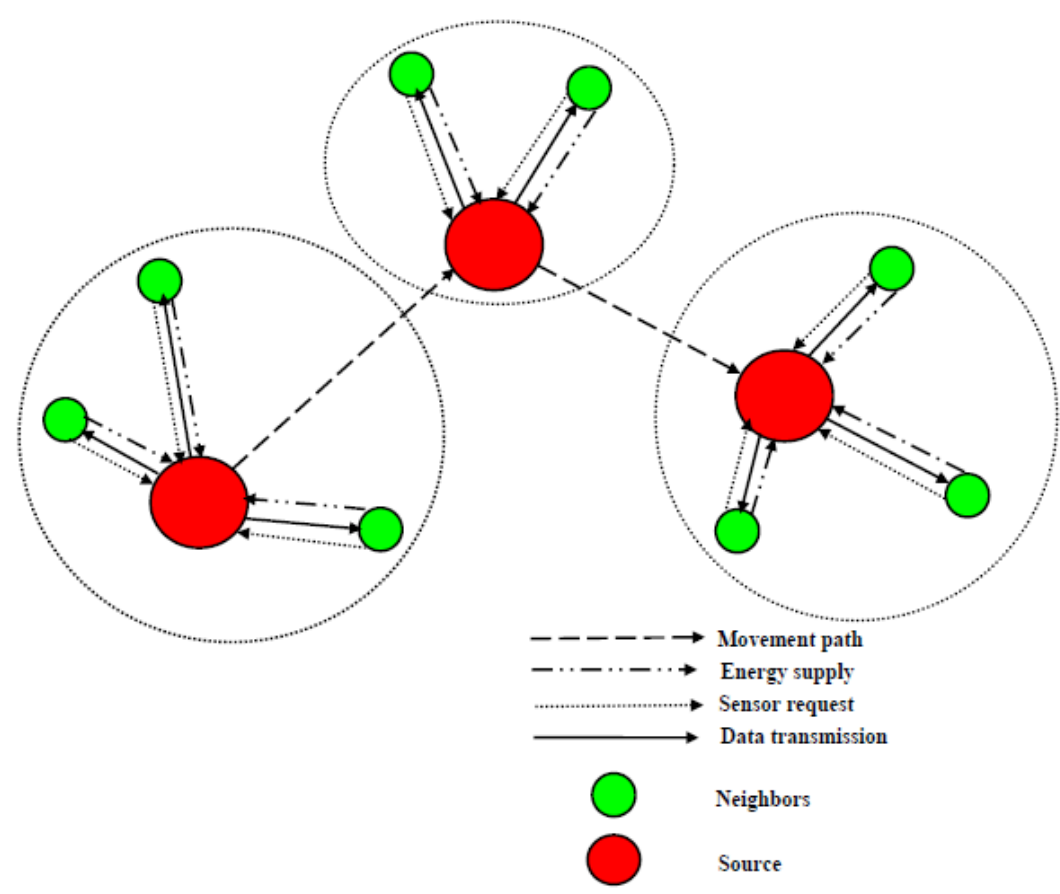

Fig. 1. sensor request and energy supply scheme

Fig. 1 shows the energy supply process between a source node and its neighbor nodes. When a source node moves, it can found a sensor communication area. Its neighbors may send sensor requests to the source node. The source node may then transmit data packets according to sensor requests. The source node consumes a considerable amount of energy by transmitting data packets. By contrast, its neighbors only receive data packets but never consume energy. In this scheme, neighbors need to supply energy to sensor areas when they 
receive data packets from the source node.

From what has been discuss, we found an energy assessment model and the condition of energy supply to establish a good scheme in supply energy with nodes.

\subsubsection{Energy assess model}

Energy consumption in opportunistic networks comes from data transmission, signal processing, and hardware operation. Particularly in data transmission, we consider energy consumption in scan, data transmission, and received data. These factors establish some models.

(1) Energy consumption in scan shows energy consumption in scan channel with node. Each scan consumes $e_{s}$. Scan period is $T$. Energy consumption $E_{s}$ for node is

$$
E_{s}=e_{s} \times \frac{t}{T}
$$

Where $t$ shows working time with node.

(2) Energy consumption is in transmission. The sent data packet needs to consume energy. Each sent data packet must consume $e_{t} . S_{t}$ data packets are transmitted. Energy consumption in transmission $E_{t}$ is:

$$
E_{t}=e_{t} \times S_{t}
$$

(3) Energy consumption is in receiving. The condition is the same as receiving data packets by nodes. Each data packet received must consume $e_{r} . S_{r}$ data packets are transmitted. Energy consumption in transmission $E_{r}$ is:

$$
E_{r}=e_{r} \times S_{r}
$$

From Eqs. (1) to (3), energy consumption $E_{c}$ and surplus energy $E_{\text {sur }}$ are

$$
\left\{\begin{array}{l}
E_{c}=E_{t}+E_{r}+E_{s} \\
E_{\text {sur }}=E_{c a r}-E_{c}
\end{array} .\right.
$$

Energy can be calculated easily according to the established energy assessment model.

\subsubsection{Condition of energy supply}

Each node in opportunistic networks may become a source node or a neighbor. Energy supply can extend the lifetime with nodes in communication in a big data environment. Thus, the condition of energy supply is founded to deliver some energy between nodes and extend the average lifetime with nodes.

The process of supplying energy can be realized through the following steps:

1. An energy supply threshold $E_{\lim }$ is set for all of the nodes. Neighbor nodes supply energy to the source node when the carrying energy $E_{c a r} \geq E_{\lim }$. Otherwise, the neighbor nodes do nothing.

2. Neighbor nodes survey the number of effective packets after they have received the packets sent by the source node. Effective data packets are calculated as follows.

(1) Assume that source node $S$ has $n$ neighbors $Q_{n}$ to obtain energy supply, as shown in Fig. 2. 
Fig 2. shows that source node $S$ sends data packets from its neighbors after $S$ receives requests from assemble $Q_{n}$. Each neighbor surveys data packets via the XOR form, $Q_{n} \oplus P$.

$$
\left\{P \oplus Q_{i}\right\}_{\text {data }}=p_{i} 1 \leq i \leq n, p_{i} \subseteq p_{n} .
$$

$\left\{P \oplus Q_{i}\right\}_{\text {data }}$ indicates that node $Q_{i}$ and $P$ carry different data packets.

(2) After receiving XOR data packets, neighbors would compare energy supply threshold $E_{\text {lim }}$. If the carrying energy is $E_{c a r} \geq E_{\lim }$ for neighbor node, it will prepare supply energy in Eqs.(6) from source node $S$. A neighbor node $Q_{i}$ can supply energy to source node $S$.

(3)

$$
E_{Q_{i} \longrightarrow S}=\sum_{Q_{i} \oplus S} e_{p_{i}}
$$

$\sum_{Q_{i} \oplus S} e_{p_{i}}$ represents the supplementary data packets of all XOR packets for node $Q_{i}$. $\sum_{Q_{i} \oplus S} e_{p_{i}}=p_{i} \times e_{t}$.



Fig. 2. XOR(exclusive-OR) data packets are transmitted from source node $S$ to its neighbors assemble $Q_{n}$

(4) Through the aforementioned methods, the energy supply of all neighbors of node $S$ and $Q_{n}$ is surveyed in Fig. 3. 


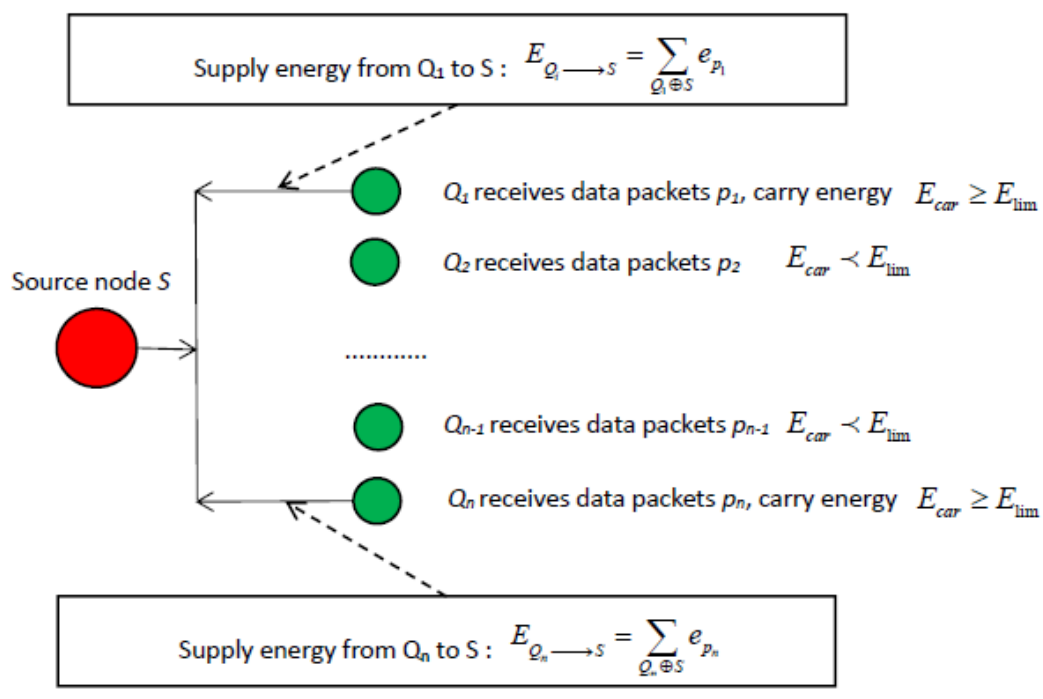

Fig. 3. Energy supply from neighbors to source node

Fig. 3 shows that each node with the conditions of satisfaction $E_{c a r} \geq E_{\lim }$ can be supply energy to source node. The total supply energy is:

$$
E_{\text {sup }}=\sum_{j \subseteq N} \sum_{Q_{i} \oplus S} e_{p_{i j}}
$$

$j \subseteq N$ shows the number of nodes, which are the conditions of satisfaction $E_{\text {car }} \geq E_{\text {lim }}$.

(4) The best and worst cases of the energy supply strategy for a source node are as follows.

Best case: The carrying energy of all the neighbor nodes $Q_{n}$ satisfies $E_{c a r} \geq E_{\lim }$. Node $S$ obtains a full energy supply.

Worst case: The carrying energy of all the neighbor nodes $Q_{n}$ satisfies $E_{c a r}<E_{\text {lim }}$. Node $S$ cannot acquire energy supply and becomes the source node of the Spray and Wait algorithm.

The worst case seldom occurs given the large number of nodes in big data networks. Consequently, the source node involved in information transmission can acquire energy supply and avoid death.

\subsubsection{Influence between energy and lifetime with node}

Lifetime with node in communication in opportunistic networks is usually controlled by energy. The more a node extends lifetime, the more data packets can be transmitted. We provide a definition to judge influence between energy and lifetime with node.

Definition 1: Node survival rate. This item shows effective communication time rate with node. It is determined by carrying energy $E_{c a r}$, consuming energy $E_{c}$, supply energy for neighbors $E_{\text {sup }}$, and communication time $\Delta T$. Node survival rate $\delta_{s}$ is 


$$
\delta_{s}=\frac{E_{c a r}-E_{c}+E_{\text {sup }}}{\Delta T}
$$

Influence can be assessed between energy and lifetime with node according to the calculation of survival rate. When energy is lacking, neighbors can supply energy and extend lifetime for node in communication.

\subsection{Iterative process of node energy transmission}

When nodes meet in opportunistic networks, each node that receives the route sensor requests from its neighbor nodes, it plays the role of the source node. If it sends a route request to the destination node, then it will become a neighbor. The process appears to be random, wherein the node sends and receives data packets by moving.

In a big data communication environment, the maximum utilization of the carrying energy of a node can ensure effective data packets deliver between nodes and maintain the utilization efficiency of the entire network energy. Therefore, the node can ensure that the energy utilization of the network is adjusted and the carrying energy limit $E_{\lim }$ is changed.

The carrying energy limit $E_{\text {lim }}$ is determined by the average of the energy carried with the entire network nodes. That is, $E_{\lim }=\lambda \overline{E_{\text {ave }}}, 0<\lambda<1 . \overline{E_{\text {ave }}}$ denotes the mean carried energy of all network nodes. To calculate the limit, the carried energy values of each node at different time segments are required.

In opportunistic networks, nodes transmit and receive data packets by moving randomly and iteratively. To calculate the energy value at a particular moment, the energy carried by a node during a prior period should be determined. Thus, node energy can be obtained by recursion according to the iterative process. This process is shown in Fig. 4.

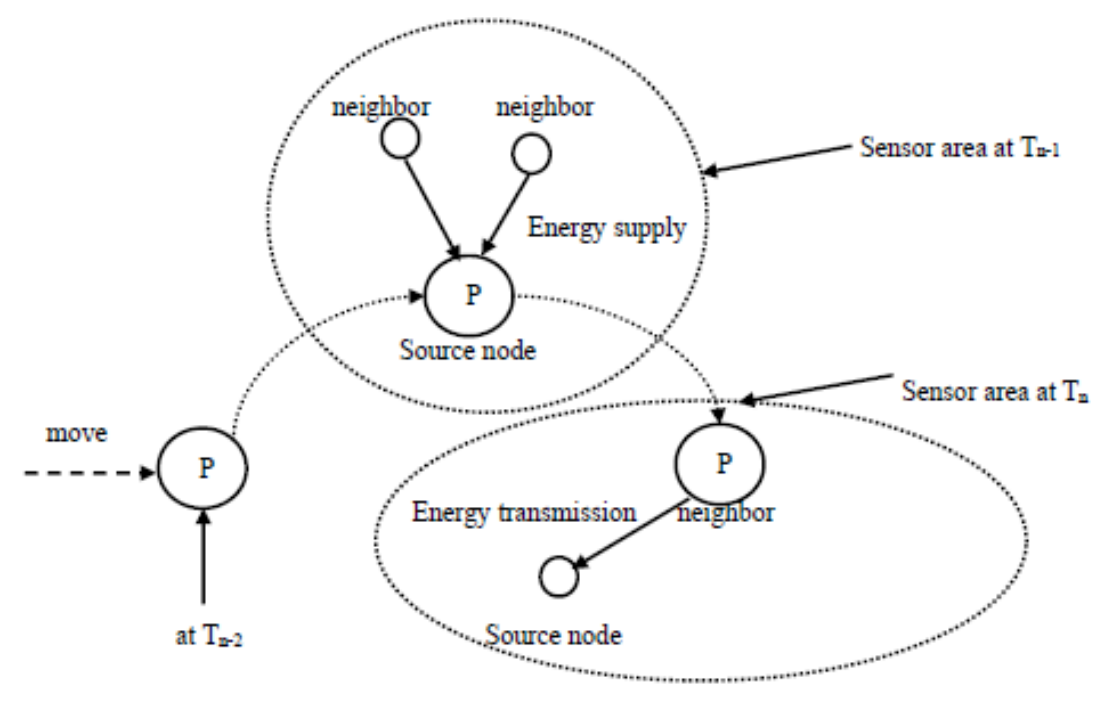

Fig. 4. Recursion of energy transmission

The model lists the recursion of the energy transmission of single node $P$. At time $\mathrm{T}_{0}$, node $P$ carries energy $E\left(\mathrm{~T}_{0}\right)=E_{0}$. 
At time T1, the energy consumption of $P$ is $\Delta E\left(\mathrm{~T}_{1}\right)$. Node $P$ at time $\mathrm{T}_{1}$ carries energy.

$$
E\left(\mathrm{~T}_{1}\right)=E\left(\mathrm{~T}_{0}\right)-\Delta E\left(\mathrm{~T}_{1}\right)
$$

The following discussion on the energy consumption of the source node and the neighbor nodes is illustrated in Fig. 4.

At time $\mathrm{T}_{\mathrm{n}-1}$, node $P$ sends data packets to its neighbors as the source node. Assuming that $P$ has $m$ neighbors, the energy consumption of sending data packets at this time is

$$
E_{\text {send }}\left(\mathrm{T}_{n-1}\right)=e_{1}+e_{2}+\cdots \cdots+e_{m}=\sum_{i=1}^{m} e_{i}
$$

At time $\mathrm{T}_{\mathrm{n}-1}, k$ neighbors can supply energy to node $P$ among $m$ neighbors. Consequently, the energy supply is

$$
E_{\text {sup }}\left(\mathrm{T}_{n-1}\right)=\sum_{k \subset m} e_{k} \quad e_{k} \subseteq\left\{e_{1}, e_{2} \cdots \cdots e_{m}\right\}
$$

At time $\mathrm{T}_{\mathrm{n}-1}$, the energy consumption of node $P$ is

$$
\Delta E\left(\mathrm{~T}_{n-1}\right)=E_{\text {send }}\left(\mathrm{T}_{n-1}\right)-E_{\text {sup }}\left(\mathrm{T}_{n-1}\right)
$$

According to Formula (7), the carried energy of $P$ after data packet transmission at time $\mathrm{T}_{\mathrm{n}-1}$ is

$$
\begin{aligned}
E\left(\mathrm{~T}_{n-1}\right) & =E\left(\mathrm{~T}_{n-2}\right)-\Delta E\left(\mathrm{~T}_{n-1}\right) \\
& =E\left(\mathrm{~T}_{n-2}\right)-\left(E_{\text {send }}\left(\mathrm{T}_{n-1}\right)-E_{\text {sup }}\left(\mathrm{T}_{n-1}\right)\right)
\end{aligned}
$$

According to Fig. 4, node $P$, as the neighbor node, obtains packets from the source node at time $\mathrm{T}_{\mathrm{n}}$. Assume that node $P$ carries more energy than the energy limit at time $\mathrm{T}_{\mathrm{n}}$. Node $P$ transmits energy to the source node. The energy consumption at time $T_{n}$ is

$$
\Delta E\left(\mathrm{~T}_{n}\right)=E_{\text {send }}\left(\mathrm{T}_{n}\right)
$$

Based on this formula, the carried energy of node $P$ at time $T_{n}$ is

$$
\begin{aligned}
E\left(\mathrm{~T}_{n}\right) & =E\left(\mathrm{~T}_{n-1}\right)-\Delta E\left(\mathrm{~T}_{n}\right) \\
& =E\left(\mathrm{~T}_{n-2}\right)-\Delta E\left(\mathrm{~T}_{n-1}\right)-\Delta E\left(\mathrm{~T}_{n}\right)
\end{aligned}
$$

Through iteration, the carrying energy of the nodes at each $\mathrm{T}$ is obtained.

For all the nodes in opportunistic networks, the average carrying energy at time $\mathrm{T}$ is

$$
\overline{E_{\text {ave }}}=\frac{\sum_{j=1}^{N} E_{j}\left(\mathrm{~T}_{n}\right)}{N}
$$

From $E_{\lim }=\lambda \overline{E_{\text {ave }}}$, the energy limit of nodes, as well as the efficiency of the network can be improved.

In the algorithm, the energy consumption of each node exhibits a functional relationship.

$$
E_{\text {node }}=\iint f(e, p) \operatorname{ded} p
$$

That is, energy consumption is decided by the number of data packets and the energy consumed with each data packet. If each packet, which is contained in sufficient storage space, has the same length, then the nodes transmit each packet that consumes the same amount of energy. Under this condition, energy consumption is 


$$
\begin{aligned}
E_{\text {node }} & =\iint f(e, p) \operatorname{ded} p \\
& =\int C_{1} f(p)+C_{2} d p
\end{aligned}
$$

C1 and C2 show a functional constant relationship between data packet and energy consumed.

\section{Algorithm design and simulation experiment}

\subsection{Design of the sensor request energy supply algorithm}

In Section 3, a sensor request energy supply scheme for big data opportunistic networks is proposed. Based on this scheme, the algorithm will be established as follows:

Table 1. sensor request energy supply algorithm

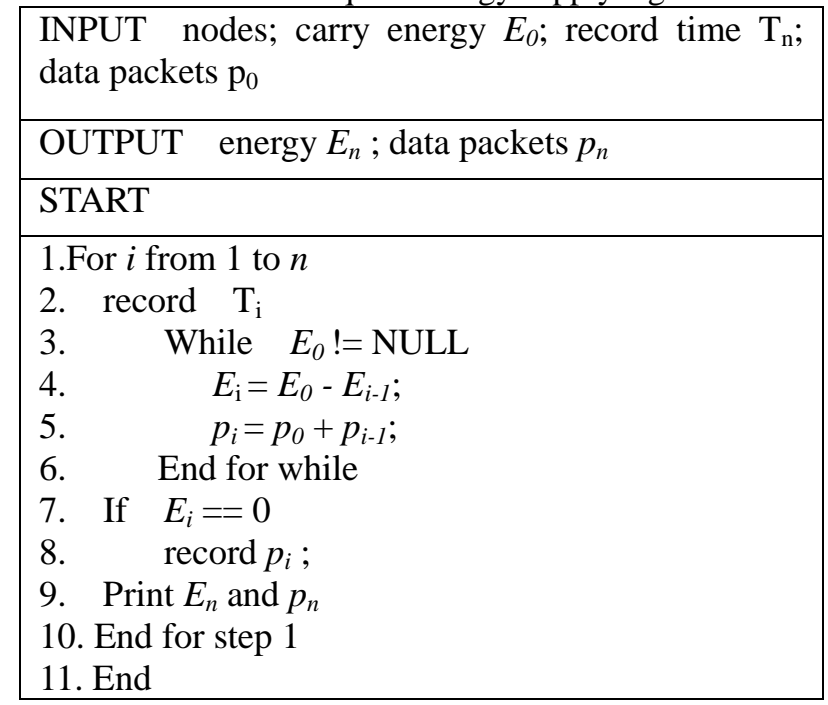

In Table 1, the time complexity with data packets is $\mathrm{O}\left(\mathrm{n}^{2}\right)$. Because of two iteration processes: a self-node transmission and a transmission that moves space and meets at different times. The time complexity with energy is also $\mathrm{O}\left(\mathrm{n}^{2}\right)$, as decided by the processing of data packets. The next step is simulation.

\subsection{Simulation}

The simulation adopts the simulator Opportunistic Networking Environment (ONE) [10] and its extended model in the opportunistic networks to compare energy consumption in these algorithms. Parameters are set based on the random model in opportunistic networks. The parameters are set in Table 2. 
Table 2. Simulation parameters

\begin{tabular}{cc}
\hline Parameter & Value \\
\hline Simulation time & 12 hours \\
Area in networks & $4500^{*} 3400 \mathrm{~m}^{2}$ \\
Communication method & Bluetooth \\
Transmission pattern & Broadcast \\
Transmit area & $10 \mathrm{~m}^{2}$ \\
Initial data packet & 10 \\
Frequency of data packet & $25-35 \mathrm{~s}$ \\
Number of nodes & $50-400$ \\
Size of data packet & $100-200 \mathrm{~KB}$ \\
Speed of transmitting & $150 \mathrm{k} \mathrm{bit} / \mathrm{s}$ \\
Speed of node movement & $0.5-1.5 \mathrm{~m} / \mathrm{s}$ \\
Cache & $5 \mathrm{MB}$ \\
Transmission pattern of nodes & $50 c i a l ~ m o d e l$ \\
Initial energy $E_{c a r}$ & $100 \mathrm{~J}$ \\
$e_{S}$ & $2.2_{t}^{*} 10^{-6} \mathrm{~J}$ \\
$e_{r}$ & $3.5^{*} 10^{-4} \mathrm{~J}$
\end{tabular}

This simulation adopts a random node in ONE. The simulation is explained as follows.

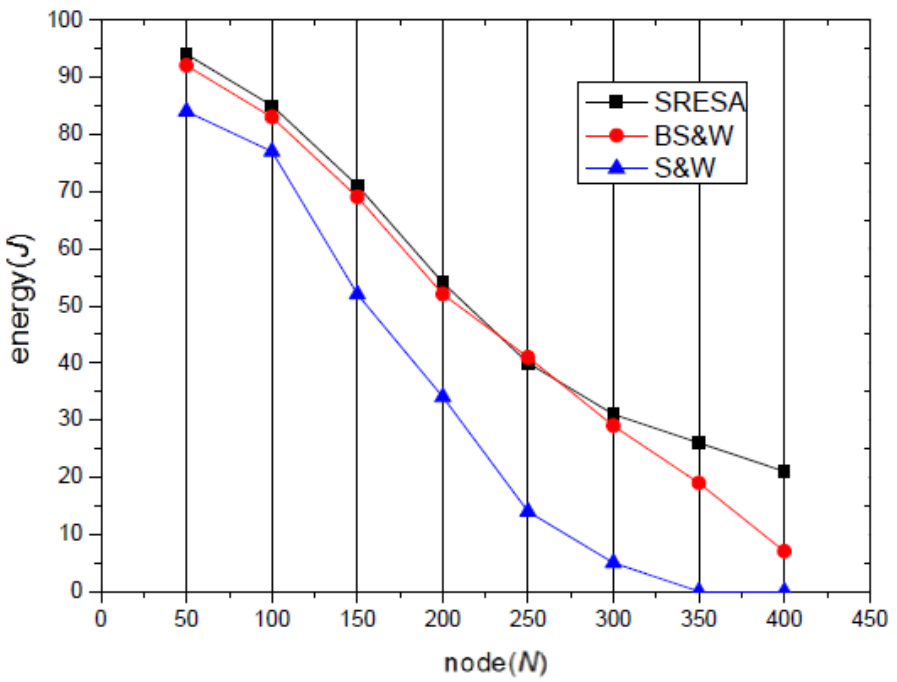

Fig. 5. Energy consumption of nodes in opportunistic networks

Fig. 5 shows the node energy consumption. In the experiment, the simulation selects an average energy level for nodes. As shown in Fig. 5, the energy threshold is 30. As the total number of nodes increases from 50 to 400, the energy of all nodes is reduced because all the 
nodes are sending packets. The carrying energy of the node has increased slightly because of the time difference between carried energy and supplied energy. In the Spray and Wait algorithm, nodes consume all energy at 345 packets, with no energy left to supply the nodes. Consequently, nodes in the Spray and Wait algorithm cannot participate in communication. At the same time, the Binary Spray and Wait algorithm and SRESA still work. When the nodes reach 400, the carrying energy in SRESA is more than that in the Binary Spray and Wait algorithm because energy has been supplied. Hence, the nodes in SRESA can transmit data packets for a long time. As shown in Fig. 5, nodes can participate in communication constantly, and no node has died with an energy supplement. By contrast, nodes without an energy supply eventually die because of energy consumption.



Fig. 6. The energy limit control of node

Fig. 6 indicates the change in energy limit of nodes and its influence on data packet transmission. In this experiment, SRESA is adopted to send data packets and the energy limit is $10 \%, 20 \%$, and 30\%. As shown in Fig. 6, as the number of nodes increases from 50 to 200, the gap between the number of nodes that are sending data packets is smaller than receiving when node on average is within the different limit. When the number of nodes is greater than 250, the limit for nodes that are sending data packets changes because in the three simulations, different nodes are randomly encountered. However, when the number of nodes increases, the data packet transmitted under a different limit increases. When the number of nodes is 400 , the most number of data packets in transmission is limited at $10 \%$, whereas it is limited at $30 \%$ when the number of data packets is the least. This finding demonstrates that data packets are under control, and that the death of a node caused by excessive sending is avoided when a limit protection is set. When the limit is low, the sending data packets are numerous and the active degrees of nodes are high. 


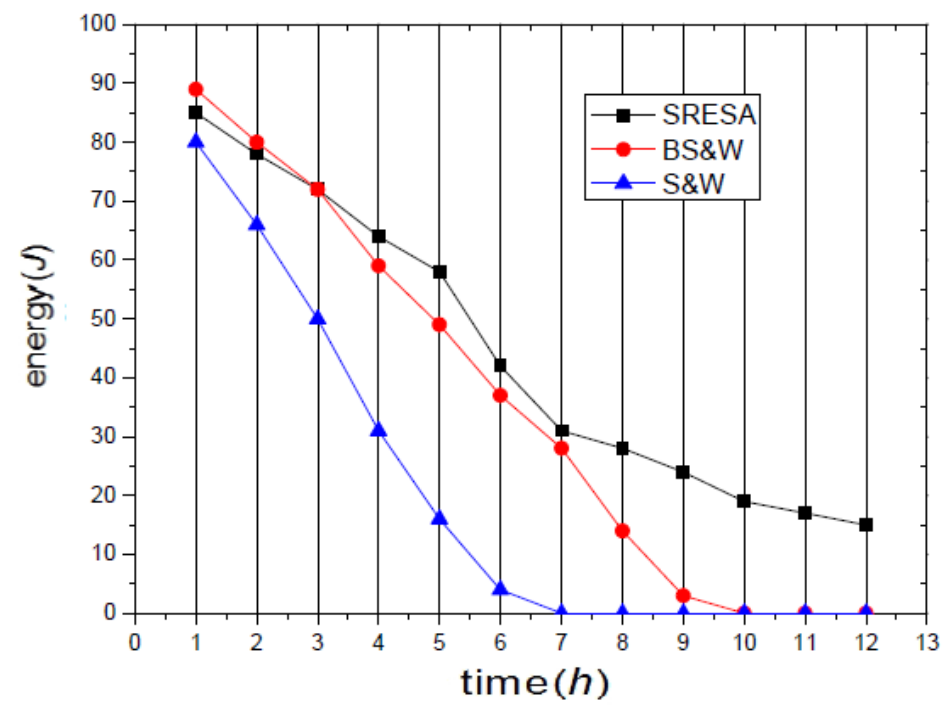

Fig. 7. Energy consumption of node at different time periods

Fig. 7 shows varying carrying energy of the source node at different simulation periods. The experiment is simulated on the map with an acceleration of 1:100. The energy limit of SRESA is set to 30\%. As shown in Fig. 7, the energy of the source node is reduced, which indicates that it is sending data packets. In SRESA, the energy of the source node is added in less than 5 hour, and carrying energy is slightly increased. When the node has transmitted data packets at 7 hour, it loses its communication capability in the Spray and Wait algorithm because of energy depletion. At 10 hour, nodes have consumed energy in the Binary Spray and Wait algorithm and cannot join in communication. In the SRESA algorithm, nodes can keep communicating without resulting in death because of the continuous energy supply.

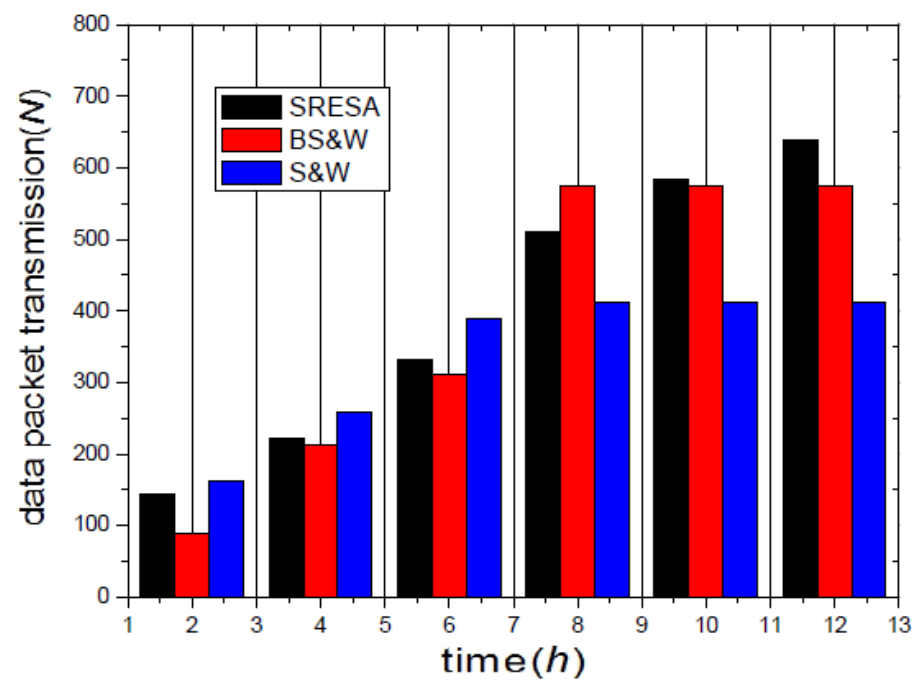

Fig. 8. Data packets transmission of node 
Fig. 8 shows the packet transmission situation of the source node. After 6 hour, nodes are still sending data packets, while the number of packets increases. However, in the Spray and Wait algorithm, node energy is used up after 8 hour. Therefore, nodes only sent approximately 410 packets. In the Binary Spray and Wait algorithm, nodes sent only approximately 580 packets, after over 9 hour because energy has been consumed. In the SRESA algorithm, the node can continue sending data packets and participate in communication because of its energy supply. After 12 hour, the node has sent over 630 data packets.

Figs. 7 and 8 show that the number of packets sent by nodes increases with increasing time, along with energy consumption. Nodes with an energy supply can maintain communication and transmit data packets, which is helpful in improving the network performance.

Table 3 and Fig. 9 record surplus energy for nodes that are transmitted in simulation. All of the nodes are divided into four groups and are indexed c0-c99, t0-t99, w0-w99, and r0-r99 in the map. Surplus energy is recorded per hour by each one hundred on average. Surplus energy for three algorithms is shown in Table 3.

They show that surplus energy in Spray and Wait algorithm is rapidly consumed. All energy is consumed in eight hours. Binary spray and wait algorithm is better than spray and wait algorithm. Node assemble c0-c99 has 0.11 energy in 12 hours. Other assembles consumed their energy for more than 10 hours. SRESA is the best algorithm. Surplus energy is approximately 20 at the end of simulation time. Thus, energy supply can extend lifetime for nodes in communication.

Table 3. Surplus energy in record nodes for simulation time

\begin{tabular}{|c|c|c|c|c|c|c|c|c|c|c|c|c|c|}
\hline \multicolumn{14}{|c|}{ Simulation time } \\
\hline \multicolumn{2}{|c|}{ Node number } & 1 & 2 & 3 & 4 & 5 & 6 & 7 & 8 & 9 & 10 & 11 & 12 \\
\hline \multirow{3}{*}{$\begin{array}{l}\text { c0- } \\
\text { c99 }\end{array}$} & S\&W & 81.46 & 67.12 & 46.31 & 33.69 & 21.22 & 11.45 & 5.11 & 0.31 & 0.01 & 0 & 0 & 0 \\
\hline & $\mathrm{BS} \& \mathrm{~W}$ & 91.44 & 81.14 & 75.12 & 60.21 & 49.13 & 40.21 & 31.22 & 18.98 & 7.14 & 3.03 & 1.17 & 0.11 \\
\hline & SRE & 87.21 & 79.12 & 76.55 & 67.55 & 60.12 & 41.89 & 37.62 & 33.58 & 30.55 & 24.56 & 19.08 & 18.23 \\
\hline \multirow{3}{*}{$\begin{array}{r}\text { t0- } \\
\text { t99 }\end{array}$} & S\&W & 78.53 & 69.33 & 53.17 & 31.55 & 23.12 & 7.34 & 0.96 & 0 & 0 & 0 & 0 & 0 \\
\hline & BS\&W & $\begin{array}{l}89.21 \\
\end{array}$ & 80.12 & 73.11 & 61.44 & 51.88 & 38.54 & 29.87 & 17.12 & 3.12 & 1.21 & 0.06 & 0 \\
\hline & SRE & 85.33 & 80.14 & 77.28 & 64.21 & 59.55 & 39.99 & 34.45 & 32.74 & 29.16 & 21.47 & 17.39 & 15.44 \\
\hline \multirow{3}{*}{$\begin{array}{l}\text { w0- } \\
\text { w99 }\end{array}$} & S\&W & 79.12 & 62.79 & 52.89 & 26.17 & 14.11 & 6.89 & 0 & 0 & 0 & 0 & 0 & 0 \\
\hline & BS\&W & 90.11 & 82.14 & 74.89 & 57.64 & 46.73 & 32.55 & 25.43 & 10.79 & 1.14 & 0 & 0 & 0 \\
\hline & SRE & 84.86 & 81.22 & 75.46 & 63.78 & 57.19 & 37.55 & 32.44 & 29.58 & 27.56 & 18.05 & 14.34 & 11.51 \\
\hline \multirow{3}{*}{$\begin{array}{r}\text { r0- } \\
\text { r99 }\end{array}$} & S\&W & 79.88 & 64.14 & 47.86 & 30.32 & 16.87 & 4.21 & 0 & 0 & 0 & 0 & 0 & 0 \\
\hline & BS\&W & 90.17 & 78.47 & 72.89 & 56.21 & 48.82 & 34.81 & 26.17 & 11.22 & 2.21 & 0.41 & 0 & 0 \\
\hline & SRE & 86.79 & 77.91 & 73.48 & 62.92 & 56.38 & 36.28 & 31.48 & 28.47 & 26.81 & 16.14 & 12.07 & 10.39 \\
\hline
\end{tabular}



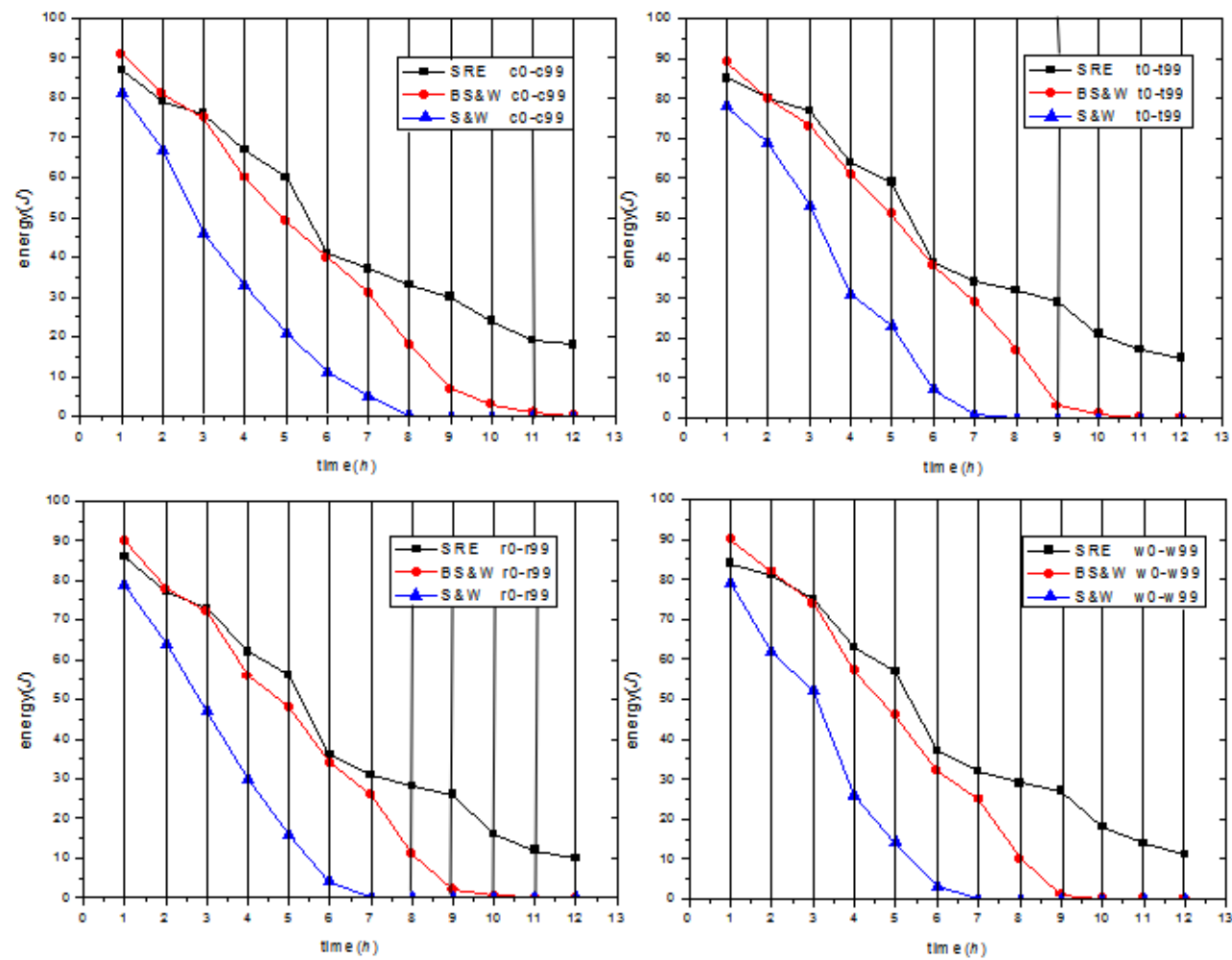

Fig. 9. Surplus energy in different groups for simulation time

\section{Conclusion}

An energy transfer and supply scheme for nodes is proposed in this study to improve communication in an opportunistic network. The scheme aims to avoid the death of nodes when energy supply runs dry. In future endeavors, we will study energy supply and energy distribution optimization to help improve the communication capability of network nodes.

\section{References}

[1] Hu Y, Liu A., "An efficient heuristic subtraction deployment strategy to guarantee quality of event detection for WSNs,” Computer Journal, vol. 58, no. 8, pp. 1747-1762, August. 2015. Article (CrossRef Link)

[2] Conti M, Kumar M., "Opportunities in opportunistic computing,” Computer, vol. 43, no. 1, pp. 42-50, January. 2010. Article (CrossRef Link)

[3] Pelusi L, Passarella A, Conti M., "Opportunistic networking: data forwarding in disconnected mobile ad hoc networks,” Communications Magazine, IEEE, vol. 44, no. 11, pp. 134-141, November. 2006. Article (CrossRef Link)

[4] Ristanovic N, Theodorakopoulos G, Le Boudec J Y., "Traps and pitfalls of using contact traces in performance studies of opportunistic networks," in Proc. of IEEE Conf. On INFOCOM, pp.1377-1385, February 11-12, 2012. Article (CrossRef Link)

[5] Wang Z, Chen Y, Li C., “A New Loop-Free Proactive Source Routing Scheme for Opportunistic Data Forwarding in Wireless Networks,” Communications Letters, IEEE, vol. 15, no. 11, pp. 1184-1186, November, 2011. Article (CrossRef Link) 
[6] Yomo H, De Carvalho E., “A CSI estimation method for wireless relay network," Communications Letters, IEEE, vol. 11, no. 16, pp. 480-482, June, 2007. Article (CrossRef Link)

[7] Liu C H, Xue F., "Network coding for two-way relaying: rate region, sum rate and opportunistic scheduling," in Proc. of IEEE Conf. On Communications, pp.1044-1049, May 19-23, 2008. Article (CrossRef Link)

[8] Song L, Kotz D F., "Evaluating opportunistic routing protocols with large realistic contact traces," in Proc. of the second ACM workshop on Challenged networks. ACM, pp.35-42, September 14, 2007. Article (CrossRef Link)

[9] Conti M, Giordano S, May M, et al., "From opportunistic networks to opportunistic computing," Communications Magazine, IEEE, vol. 48, no. 9, pp. 126-139, September, 2010. Article (CrossRef Link)

[10] Spyropoulos T, Psounis K, Raghavendra C S., "Spray and wait: an efficient routing scheme for intermittently connected mobile networks," in Proc. of ACM SIGCOMM workshop on Delay-tolerant networking, ACM, pp.252-259, May 11-14, New York, 2005. Article (CrossRef Link)

[11] Xue J, Fan X, Cao Y, et al., "Spray and wait routing based on average delivery probability in delay tolerant network," in Proc. of Networks Security, Wireless Communications and Trusted Computing, 2009. NSWCTC'09. International Conference, IEEE. pp. 500-502, April 25-26, 2009. Article (CrossRef Link)

[12] Aliotta J M, Pereira M, Johnson K W, et al., "Microvesicle entry into marrow cells mediates tissue-specific changes in mRNA by direct delivery of mRNA and induction of transcription," Experimental hematology, vol. 38, no.3, pp. 233-245,March, 2012. Article (CrossRef Link)

[13] Lokhov A Y, Mézard M, Ohta H, et al., "Inferring the origin of an epidemic with a dynamic message-passing algorithm,” Physical Review E, vol. 90, no.1, pp.012801-012801, January 2014. Article (CrossRef Link)

[14] Wang G, Wang B, Gao Y., "Dynamic spray and wait routing algorithm with quality of node in delay tolerant network," in Proc. of Communications and Mobile Computing (CMC), 2010 International Conference on. IEEE. pp.452-456, April 12-14, 2010. Article (CrossRef Link)

[15] Misra S, Ojha T, Mondal A., "Game-Theoretic Topology Controlfor Opportunistic Localization in Sparse Underwater Sensor Networks,” IEEE Transactions on Mobile Computing, vol.14, pp.990-1003, May, 2015. Article (CrossRef Link)

[16] Leguay J, Friedman T, Conan V., "DTN routing in a mobility pattern space," in Proc. of ACM SIGCOMM workshop on Delay-tolerant networking, ACM, pp.276-283, June 22-24, 2005. Article (CrossRef Link)

[17]Burgess J, Gallagher B, Jensen D, et al., "MaxProp: Routing for Vehicle-Based Disruption-Tolerant Networks,” in Proc. of INFOCOM, pp.1-11, April 23-29, 2006. Article (CrossRef Link)

[18] Burns B, Brock O, Levine B N., "MORA routing and capacity building in disruption-tolerant networks,” Ad hoc networks, vol.6, no.4, pp.600-620, April, 2008. Article (CrossRef Link)

[19] Estrin D., "Small data, where n=me," Communications of the ACM, vol.57, no.4, pp. 32-34, April, 2014. Article (CrossRef Link)

[20] Edalat N, Motani M., "Energy-aware task allocation for energy harvesting sensor networks," EURASIP Journal on Wireless Communications and Networking, vol.27, no 1, pp.1-14, January, 2016. Article (CrossRef Link)

[21]Dong M, Ota K, et al., "Joint Optimization of Lifetime and Transport Delay under Reliability Con straint Wireless Sensor Networks,” IEEE Transactions on Parallel and Distributed Systems, vol.27, no.1, pp.225-236, January, 2016. Article (CrossRef Link) 


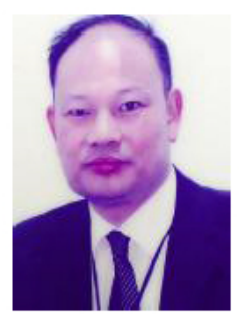

Zhi-gang Chen received the BE, the MS and $\mathrm{PhD}$ from Central South University in China in 1984, 1987 and 1998. He is currently a Professor, Supervisor of PhD and Dean of School of software, Central South University. He is also director and advanced member of China Computer Federation (CCF), and member of pervasive computing committee of CCF. His research interests cover the general area of cluster computing, parallel and distributed system, computer security, wireless networks.



Jia WU received his BS Degree from Gui Zhou University in China 2006, and he received his MS Degree from Yun Nan Uniersity in China 2009. He is a $\mathrm{PhD}$ candidate in software engineering at Central South University. He is system analyst and advanced member of China Computer Federation (CCF). His research interests are wireless networks, and big data research. 